\title{
Height of elevated fetal buttock for prediction of successful external cephalic version
}

Jun Yi Lee, MD¹, Yeorae Kim, MD², In Sook Sohn, MD, PhD', You Jung Han, MD, PhD², Jin Hoon Chung, MD, PhD ${ }^{3}$, Moon Young Kim, MD, PhD ${ }^{3}$, Min Hyoung Kim, MD, PhD ${ }^{4}$, Hyun-Mee Ryu, MD, PhD ${ }^{5}$, SungHong Joo, MD, PhD ${ }^{6}$, Jung Yeol Han, MD, PhD ${ }^{6}$

Department of Obstetrics and Gynecology, ${ }^{1}$ KonKuk University Medical Center, Konkuk University School of Medicine, Seoul, ${ }^{2}$ Seoul National University Bundang Hospital, Seoul National University College of Medicine, Seongnam, ${ }^{3} \mathrm{CHA}$ Gangnam Medical Center, CHA University, Seoul, ${ }^{4}$ Gangseo Mizmedi Hospital, Seoul, ${ }^{5} \mathrm{CHA}$ Bundang Medical Center, CHA University, Seongnam, ${ }^{6}$ National Medical Center, Seoul, Korea

\section{Objective}

To increase the rate of successful external cephalic version (ECV) and to minimize the complications, it is important to identify the predictors of success. Therefore, the purpose of this study was to investigate whether the height of the elevated fetal buttock (HOB) is a valuable predictor of successful ECV or not.

\section{Methods}

This prospective study was conducted from August 2016 to June 2018. A total of 139 pregnant women with breech presentation were enrolled in the study. HOB from the maternal pubic symphysis was measured on ultrasonography. The predictability and cut-off value of HOB for successful ECV were evaluated.

\section{Results}

Among the 139 patients, $114(82 \%)$ had successful ECV. The adjusted odds ratio for multiparity, amniotic fluid index (AFI) $>14 \mathrm{~cm}$, and $\mathrm{HOB}>7.8 \mathrm{~cm}$ were 10.80 (95\% confidence interval $[\mathrm{Cl}], 1.57-74.94), 5.26(95 \% \mathrm{Cl}, 1.06-26.19)$, and $10.50(95 \% \mathrm{Cl}, 1.03-107.12)$, respectively. Areas under the curve (AUCs) for $\mathrm{AFI}, \mathrm{HOB}$, and parity were $0.66(95 \% \mathrm{Cl}$, $0.54-0.78), 0.74(95 \% \mathrm{Cl}, 0.64-0.85)$, and $0.69(95 \% \mathrm{Cl}, 0.62-0.76)$, respectively. HOB had the largest AUC, but there were no significant differences among the AUCs of other factors. The cut-off value of HOB was $6 \mathrm{~cm}$.

\section{Conclusion}

This study showed that the AUC of $\mathrm{HOB}$ was greater than that of parity and AFI, although it was not statistically significant. As HOB is a noninvasive and comprehensive marker to predict successful ECV, consideration of HOB would be helpful before conducting ECV. Further studies are needed.

Keywords: Breech presentation; External cephalic version; Predictive value

\section{Introduction}

Breech presentation occurs in approximately $3-4 \%$ of women with singleton pregnancies [1]. As breech presentation is risky for maintenance of pregnancy and delivery, practitioners perform several measures to prevent the adverse effects.

External cephalic version (ECV) is a procedure performed to change the fetal presentation from breech to cephalic by exerting pressure through the maternal abdominal wall [2]. ECV reduces the rate of cesarean section in breech presentation in the late gestational period and the risk of fetal morbidity and mortality [3].
Received: 2019.02.22. Revised: 2019.06.03. Accepted: 2019.06.21. Corresponding author: Jung Yeol Han, MD, PhD

Department of Obstetrics and Gynecology, National Medical Center, 245 Eulji-ro, Jung-gu, Seoul 04564, Korea

E-mail: hanjungyeol055@gmail.com

https://orcid.org/0000-0001-5611-2392

Articles published in Obstet Gynecol Sci are open-access, distributed under the terms of the Creative Commons Attribution Non-Commercial License (http://creativecommons. org/licenses/by-nc/3.0/) which permits unrestricted non-commercial use, distribution, and reproduction in any medium, provided the original work is properly cited.

Copyright $\odot 2020$ Korean Society of Obstetrics and Gynecology 


\section{Obstetrics \& Gynecology Science}

Vol. 63, No. 1, 2020

In 2012 , the rate of cesarean delivery was $32.8 \%$ in the United States of America and 36.0\% in Korea [2]. In 2018, the American College of Obstetricians and Gynecologists published recommendations reaffirming the existing guidelines that obstetricians should offer and perform ECV if possible $[4,5]$. The success rate of ECV varies from $30 \%$ to $80 \%$ $[2,4]$. However, ECV is associated with various complications, such as placental abruption, fetal-maternal hemorrhage, fracture of the fetal femur, prolonged fetal tachycardia, and sinusoidal pattern of the fetal heart rate [5].

Factors associated with successful ECV should be detected to increase the success and uptake rate of ECV and to minimize complications. The known factors associated with successful ECV are maternal height, weight, body mass index (BMI), parity, placental location, estimated birth weight (EBW), and amniotic fluid index (AFI). Among these, the predictors of successful ECV are parity and AFI [4,6]. Additionally, some clinical factors are associated with successful ECV, such as the surgeon's experience and dexterity with the procedure [7].

However, prediction of the success of ECV is limited using the primary factors, such as maternal BMI, EBW, breech type, $A F I$, and parity. Secondary markers can comprehensively reflect the basic and primary factors and are useful for the objective prediction of successful ECV. Additionally, a condition for successful ECV is free-floating elevation of the fetal buttocks from the maternal pelvis by the surgeon. Therefore, we determined the height of the elevated fetal buttock (HOB) by measuring the distance between the pubic symphysis, confirmed on ultrasonography, and the fetal buttock elevated manually by the surgeon (Fig. 1). If HOB were the most reliable factor for successful ECV, complications, such as fetal bradycardia and PROM, because of failed ECV would be reduced by applying HOB before ECV is performed. Therefore, the purpose of this study was to evaluate $\mathrm{HOB}$ as a marker of successful ECV.

\section{Materials and methods}

The study was prospectively designed and conducted from
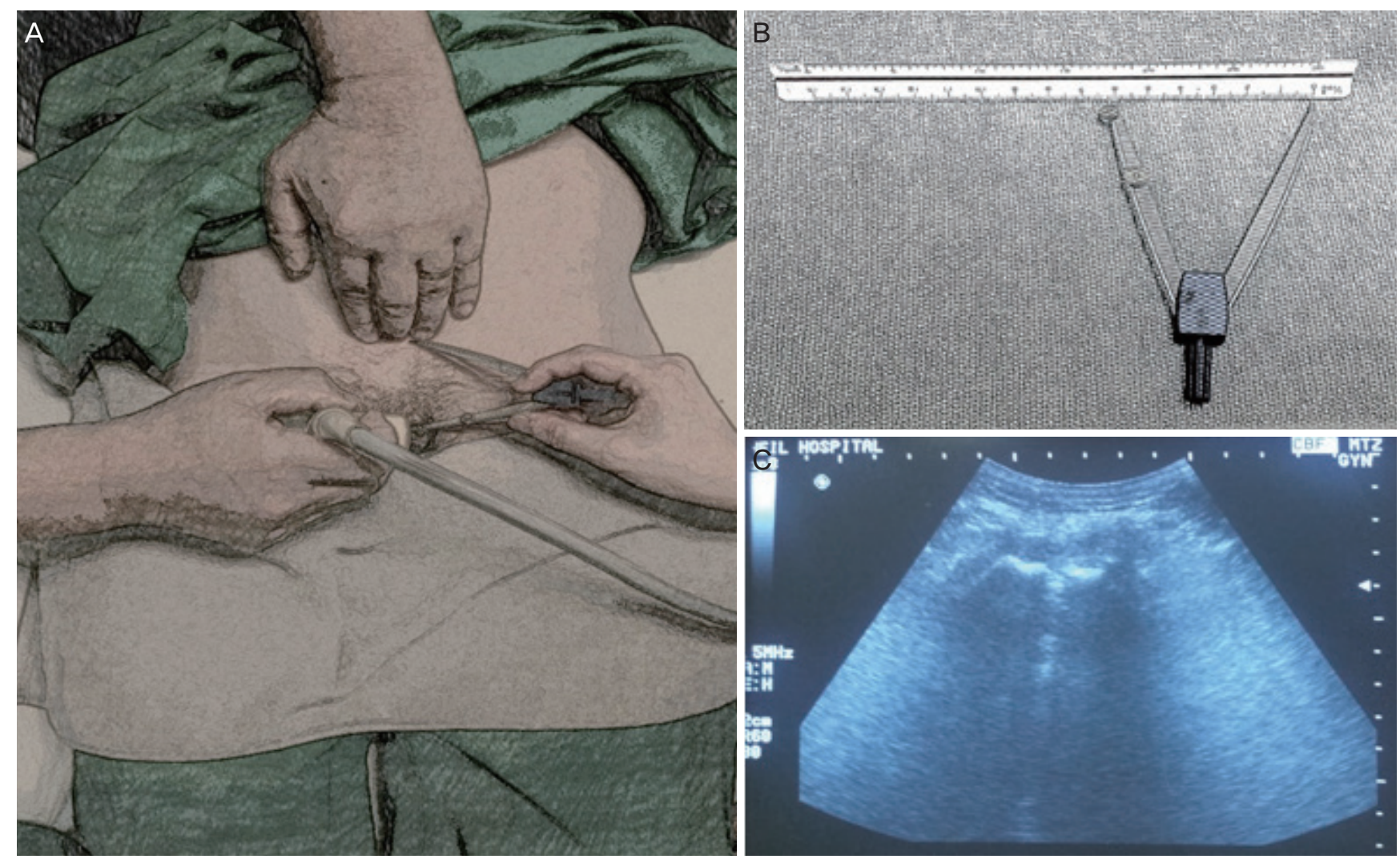

Fig. 1. Measurement of height of the elevated fetal buttock (HOB). (A) HOB by an operator's hand was measured from the maternal symphysis pubis under ultrasonography. (B) A compass and a ruler for measurement. (C) Symphysis pubis seen on ultrasonography. 


\section{Obstetrics \& Gynecology Science}

Jun Yi Lee, et al. External cephalic version

August 2016 to June 2018.

A total of 139 pregnant women with breech presentation were enrolled in the study. No participant presented with contraindications for ECV, such as preeclampsia, multiple pregnancy, antepartum hemorrhage, oligohydramnios, uteroplacental insufficiency, intrauterine growth retardation, or non-reassuring fetal monitoring pattern. Most ECVs were performed with ritodrine tocolysis, under epidural analgesia, following at least 30 minutes of fetal monitoring to ensure a normal fetal heart rate and uterine contractions in the deliv- ery unit at the Cheil General Hospital \& Women's Healthcare Center. However, some pregnant women did not consent to ritodrine tocolysis or epidural analgesia.

The ECV team comprised of 4 members, including a main supervisor (clinical surgeon), a sonographer (resident), and 2 helpers, including one nurse. Before initiating $E C V, H O B$ was measured by the surgeon and a helper using a compass and a ruler. The surgeon pushed the fetal buttock upward toward the uterine fundus manually, and the resident measured $\mathrm{HOB}$ from the maternal pubic symphysis (Fig. 1). Further, EBW,

Table 1. Demographics and prediction factors for successful external cephalic version (ECV)

\begin{tabular}{|c|c|c|c|}
\hline Characteristics & Successful ECV $(n=114)$ & Failed ECV $(n=25)$ & $P$-value \\
\hline Maternal age (yr) & $34.0 \pm 4.2$ & $33.1 \pm 4.1$ & 0.335 \\
\hline Parity & 0.54 & 0.08 & 0.001 \\
\hline Gestational age (wk) & $37.47 \pm 0.69$ & $37.44 \pm 0.64$ & 0.836 \\
\hline Estimated birth weight (g) & $2,907 \pm 317$ & $2,857 \pm 237$ & 0.469 \\
\hline Maternal height (cm) & $162.46 \pm 5.33$ & $164.12 \pm 5.13$ & 0.157 \\
\hline Maternal weight (kg) & $67.39 \pm 9.03$ & $70.32 \pm 10.09$ & 0.152 \\
\hline Maternal body mass $\left(\mathrm{kg} / \mathrm{m}^{2}\right)$ & $25.47 \pm 3.18$ & $26.20 \pm 3.18$ & 0.303 \\
\hline Presence of myoma & & & 0.041 \\
\hline Yes & $2(1.8)$ & $3(12.0)$ & \\
\hline No & $112(98.2)$ & $22(88.0)$ & \\
\hline Location of placenta & & & 0.862 \\
\hline Anterior & $14(17.5)$ & $66(82.5)$ & \\
\hline Posterior & $11(18.6)$ & $48(81.4)$ & \\
\hline Breech category & & & 0.503 \\
\hline Frank & $67(58.8)$ & $16(64.0)$ & \\
\hline Complete & $28(24.6)$ & $7(28.0)$ & \\
\hline Incomplete & $19(16.7)$ & $2(8.0)$ & \\
\hline Tocolysis & & & 0.083 \\
\hline Yes & $113(99.1)$ & $23(92.0)$ & \\
\hline No & $1(0.9)$ & $2(8.0)$ & \\
\hline Uterine contraction on admission & & & 0.192 \\
\hline Regular & $5(4.4)$ & $3(12.0)$ & \\
\hline Irregular & $70(61.4)$ & $17(68.0)$ & \\
\hline None & $39(34.2)$ & $5(20.0)$ & \\
\hline Epidural analgesia & & & 0.689 \\
\hline Yes & $104(91.2)$ & $24(96.0)$ & \\
\hline No & $10(8.8)$ & $1(4.0)$ & \\
\hline Amniotic fluid Index $(\mathrm{cm})$ & $11.96 \pm 3.03$ & $10.20 \pm 2.86$ & 0.009 \\
\hline Height of elevated fetal buttock (cm) & $7.30 \pm 1.54$ & $6.20 \pm 1.10$ & 0.001 \\
\hline
\end{tabular}

Data are shown as mean \pm standard deviation or number (\%). 


\title{
Obstetrics \& Gynecology Science
}

\author{
Vol. 63 , No. 1, 2020
}

type of breech presentation, location of the placenta, presence of myoma, and AFI were checked on ultrasonography. The surgeon stood on the other side of the sonographer. In the process, if the fetus was descending or engaged too much in the pelvic cavity, a team member pushed the fetal buttock upward from the pelvic cavity for free floating of the fetus. If necessary, the other member stood on the opposite side of the surgeon and pushed the fetal head toward the direction of easy flow. The sonographer ran a probe along the shifting fetal head and checked the fetal heartbeat during each ECV trial. We tried to avoid exceeding 10 minutes for a single trial, and the maximum number of consecutive trials was limited to 3. ECV was discontinued immediately if the fetal heart rate showed a non-reassuring pattern or if the patient complained of an intolerable discomfort despite the use of epidural analgesia. Following ECV, we considered the procedure successful when the presentation showed a fetal head without umbilical cord compression or any odd positioning of extremities, such as the hands or feet placed under the fetal head. After a successful procedure, the patient was placed in the lateral decubitus position, and fetal monitoring was continued additionally for 3 hours. Ritodrine and analgesia were stopped. After confirmation of a reassuring fetal heartbeat and lack of regular contractions, the patient was discharged.

Univariate analyses were performed using the $\chi^{2}$ test or
Fisher's exact test for categorical data and the student's $t$-test for continuous data. Multivariate analyses were performed using the logistic regression. Area under the curve (AUC) was calculated using the receiver operating characteristics (ROC) curve, and the cut-off value was obtained. The cut-off value was considered as the maximum value in the Youden's index (sensitivity+specificity-1). A $P$-value $<0.05$ was considered statistically significant.

\section{Results}

Among the 139 patients who underwent ECV after $\mathrm{HOB}$ measurement, 114 (82\%) had successful ECV, while 25 (18\%) had failed ECV. There were no fetal complications, such as abnormal fetal heart rate or rupture of the amniotic membrane, during the measurement, and the patients did not complain of any discomfort.

The demographic predictors of successful ECV, such as maternal age, gestational age, EBW, BMI, location of the placenta, breech category, tocolysis, uterine contraction on admission, and epidural analgesia, were not significantly different between the successful and failed group. The statistically significant variables associated with successful ECV were parity $(P=0.001)$, presence of myoma $(P=0.041)$, AFI $(P=0.009)$, and $\mathrm{HOB}(P=0.001)$ (Table 1).

Table 2. Logistic regression analysis for prediction factors of successful external cephalic version

\begin{tabular}{lcc}
\hline Characteristics & OR (95\% Cl) & P-value \\
\hline Parity & 1 & 0.016 \\
Nulliparity & $10.80(1.57-74.94)$ \\
Multiparity & 1 \\
Presence of myoma & $0.11(0.01-1.24)$ \\
No & \\
Yes & 1 \\
Amniotic fluid index & $2.23(0.75-6.60)$ \\
$\leq 10.0 \mathrm{~cm}$ (25 percentile) & $5.26(1.06-26.19)$ \\
$10.1-13.9 \mathrm{~cm}(>25-<75$ percentile) & 1 \\
$\geq 14.0 \mathrm{~cm}(75$ percentile) & 0.073 \\
Height of elevated fetal buttock & \\
$\leq 6.0 \mathrm{~cm}(25$ percentile) & $2.42(0.70-8.44)$ \\
$6.1-7.7 \mathrm{~cm}(>25-<75$ percentile) & $10.50(1.03-107.12)$ \\
$\geq 7.8 \mathrm{~cm}(75$ percentile) & \\
\hline
\end{tabular}

$\mathrm{OR}$, odds ratio; $\mathrm{Cl}$, confidence interval. 


\section{Obstetrics \& Gynecology Science}

Jun Yi Lee, et al. External cephalic version

In the multivariate logistic regression analysis, the odds ratio (OR) for multiparity was 10.80 (95\% confidence interval [CI], 1.57-74.94) times that of nulliparity; OR for AFI $>14 \mathrm{~cm}$ was $5.26(95 \% \mathrm{Cl}, 1.06-26.19)$ times that of $\mathrm{AFI}<10 \mathrm{~cm}$; and $\mathrm{OR}$ for $\mathrm{HOB}>7.8 \mathrm{~cm}$ was $10.50(95 \% \mathrm{Cl}, 1.03-107.12)$ times that of $\mathrm{HOB} \leq 6 \mathrm{~cm}$ and was statistically significant. However, OR for the presence of myoma was not statistically significant ( $P=0.073$ ) (Table 2).

AUCs for AFI, $\mathrm{HOB}$, and parity were $0.659(95 \% \mathrm{Cl}, 0.537-$ $0.780), 0.744(95 \% \mathrm{Cl}, 0.639-0.850)$, and $0.687(95 \% \mathrm{Cl}$, $0.619-0.755)$, respectively. The AUC for HOB was the largest, indicating that it had the greatest influence on the success of ECV. However, there were no statistically significant differences among the AUCs. The $P$-value for the AUC between AFI and HOB was 0.213 , between AFI and parity was 0.685 , and between $\mathrm{HOB}$ and parity was 0.348 (Fig. 2). The cut-off value of HOB was $6 \mathrm{~cm}$.

\section{Discussion}

Our study found that HOB, measured (or determined) by the supervisor (clinical surgeon), might be the most useful pre-

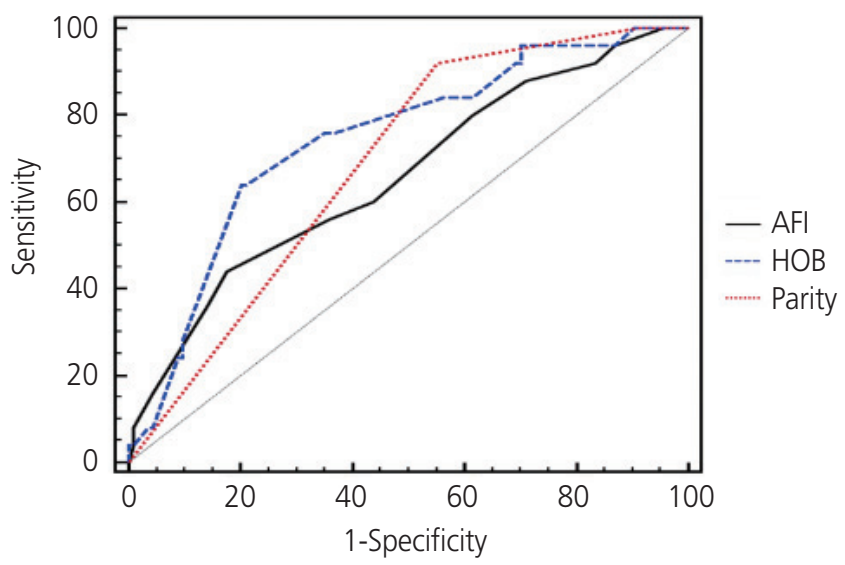

\begin{tabular}{lccc}
\hline & AUC & SE & $\mathbf{9 5 \% ~ C l}$ \\
\hline AFI & 0.659 & 0.062 & $0.537-0.780$ \\
HOB & 0.744 & 0.054 & $0.639-0.850$ \\
Parity & 0.687 & 0.035 & $0.619-0.755$ \\
\hline
\end{tabular}

Fig. 2. Receiver operating characteristics curve and AUC for validity test with $\mathrm{HOB}$, parity, AFI for prediction of successful external cephalic version. $A U C$, areas under the curve; $S E$, standard error; $\mathrm{Cl}$, confidence interval; $\mathrm{AFI}$, amniotic fluid index; $\mathrm{HOB}$, height of elevated fetal buttock. dictor of successful ECV compared to other factors such as parity and AFI. The optimal cut-off value of HOB was $6 \mathrm{~cm}$.

According to a recent study in the United States, the ECV success rate was $57.2 \%$ in the $4,981 \mathrm{ECV}$ attempts from 2003 to 2014 [4]. In this study from 2016 to 2018, the success rate was $82 \%$ in 114 patients with successful ECV among a total of 139 patients. In accordance with the previous study, 188 out of 290 ECV trials from 2013 to 2017 were successful, and the overall success rate was $64.8 \%$. The success rate was $80 \%$ in the recent 100 ECV attempts [7].

Among previous studies investigating the predictable factors for successful ECV, Burgos et al. [6] found that parity, placental location, $\mathrm{AFI}$, and the type of breech presentation were associated with successful ECV. Additionally, Kok et al. [8] found placental location as a significant factor. Mowat and Gardener [9] noted that multiparity and AFI were predictable factors for successful ECV, whereas lower AFI and nulliparity reduced the success rate of ECV. Similar to previous studies, our study also found parity and AFI to be helpful predictors of successful ECV in addition to HOB. Our study also found that the presence of myoma was a significant predictor of unsuccessful ECV in the univariate analysis; however, it was non-significant in the multivariate analysis. Yoshida et al. [10] reported that the presence of myoma is not significantly associated with ECV success or failure $(P=0.180)$.

There are some strengths of this study. To our knowledge, $\mathrm{HOB}$ is a secondary marker that can comprehensively reflect the basic and elementary factors, such as maternal age, maternal BMI, parity, EBW, AFI, type of breech, uterine tone, and location of the placenta. As this study shows, HOB is an important and reliable predictor of successful ECV. HOB measurement is noninvasive and safe for the fetus, without any complications, such as fetal distress or rupture of membranes. The procedure does not cause any maternal discomfort. In addition, this predictive factor has the largest AUC compared to other previously known significant factors, such as parity and AFI. The optimal cut-off value of $\mathrm{HOB}$, which was $6 \mathrm{~cm}$ on the ROC curve, was selected as the maximal value in the Youden's Index (sensitivity+specificity-1). When the cut-off value of HOB is over $6 \mathrm{~cm}$, the success rate can reach the maximum potential. Therefore, $\mathrm{HOB}$ is a significant marker in evaluation of ECV success rate and safe ECV.

The limitation of our study was the small sample size. More cases and further studies are necessary for generalizable findings. Although HOB had a greater AUC compared to parity 


\section{Obstetrics \& Gynecology Science}

Vol. 63, No. 1, 2020

or AFI on the ROC curve, no statistical significance was observed. Therefore, a larger sample size is necessary. Another limitation was a possible error in measuring HOB. Although the measurements were performed by the same surgeon, the assistant was different. However, compared to AFI, which is measured at 4 sites on the mother's abdomen and can be affected by fetal movement, the error in HOB measurement is likely to be lower.

We conclude that $\mathrm{HOB}$ is a noninvasive, convenient, and comprehensive marker of efficient and safe ECV and can be used for wide adoption of ECV.

\section{Conflict of interest}

No potential conflict of interest relevant to this article was reported.

\section{Ethical approval}

The study was approved by the Institutional Review Board (CGH-IRB-2017-14) of the Cheil General Hospital and Women's Healthcare Centre, Seoul, Republic of Korea.

\section{Patient consent}

The patients were informed about the possible failure and risks of the procedure, including fetal distress and emergency cesarean delivery, and their consent was obtained.

\section{References}

1. Hannah ME, Hannah WJ, Hewson SA, Hodnett ED,
Saigal S, Willan AR. Planned caesarean section versus planned vaginal birth for breech presentation at term: a randomised multicentre trial. Term Breech Trial Collaborative Group. Lancet 2000;356:1375-83.

2. Kim MY, Park MY, Kim GJ. External cephalic version experiences in Korea. Obstet Gynecol Sci 2016;59:85-90.

3. Hofmeyr GJ, Kulier R, West HM. External cephalic version for breech presentation at term. Cochrane Database Syst Rev 2015;4:CD000083.

4. Morgan ER, Hu AE, Brezak AMV, Rowley SS, Littman AJ, Hawes SE. Predictors of a successful external cephalic version: a population-based study of Washington state births. Women Birth 2019;32:e421-6.

5. ACOG Committee opinion No. 745: mode of term singleton breech delivery. Obstet Gynecol 2018;132:e60-3.

6. Burgos J, Melchor JC, Pijoán JI, Cobos P, FernándezLlebrez L, Martínez-Astorquiza T. A prospective study of the factors associated with the success rate of external cephalic version for breech presentation at term. Int J Gynaecol Obstet 2011;112:48-51.

7. Kim SY, Han JY, Chang EH, Kwak DW, Ahn HK, Ryu $\mathrm{HM}$, et al. Evaluation of the learning curve for external cephalic version using cumulative sum analysis. Obstet Gynecol Sci 2017;60:343-9.

8. Kok M, van der Steeg JW, van der Post JA, Mol BW. Prediction of success of external cephalic version after 36 weeks. Am J Perinatol 2011;28:103-10.

9. Mowat A, Gardener G. Predictors of successful external cephalic version in an Australian maternity hospital. Aust N Z J Obstet Gynaecol 2014;54:59-63.

10. Yoshida M, Matsuda H, Kawakami Y, Hasegawa Y, Yoshinaga $Y$, Hayata $E$, et al. Effectiveness of epidural anesthesia for external cephalic version (ECV). J Perinatol 2010;30:580-3. 